\title{
Seedling Resistance to Sclerotinia sclerotiorum as Expressed Across Diverse Cruciferous Species
}

Margaret Uloth, Ming Pei You, and Patrick M. Finnegan, School of Plant Biology, Faculty of Science, The University of Western Australia, Crawley, WA, 6009, Australia; Surinder S. Banga, Department of Plant Breeding and Genetics, Punjab Agricultural University, Ludhiana, 141004 Punjab, India; Huang Yi, Oil Crops Research Institute, Chinese Academy of Agricultural Sciences, Wuhan 430062, China; and Martin J. Barbetti, School of Plant Biology and The UWA Institute of Agriculture, Faculty of Science, The University of Western Australia, Crawley

Abstract

Uloth, M., You, M. P., Finnegan, P. M., Banga, S. S., Yi, H., and Barbetti, M. J. 2014. Seedling resistance to Sclerotinia sclerotiorum as expressed \begin{abstract}
across diverse cruciferous species. Plant Dis. 98:184-190.
Sclerotinia stem rot, caused by Sclerotinia sclerotiorum, is a serious disease of many cruciferous crops and frequently poses a threat to the sustainable and profitable production of these crops worldwide. Differences in seedling resistance to $S$. sclerotiorum across 46 diverse cruciferous genotypes from 12 different species were assessed by comparing the extent of pathogenesis on inoculated cotyledons under controlled conditions. Selections of Brassica carinata, B. incana, B. juncea, $B$. napus, and $B$. napus introgressed with $B$. carinata, $B$. nigra, $B$. oleracea, B. rapa var. rosularis, B. rapa var. chinensis, B. tournefortii, Raphanus raphanistrum, $R$. sativus, and Sinapis arvensis were tested. The average size of lesions on cotyledons $48 \mathrm{~h}$ post inoculation varied from 0.8 to $7.3 \mathrm{~mm}$. The three most resistant genotypes with the smallest lesions were all from B. oleracea (viz., B. oleracea var. italica 'Prophet' and B. oleracea var. capitata 'Burton' and 'Beverly Hills'). Representatives of $R$. raphanistrum, $S$. arvensis, B. juncea, and $B$. carinata were the most susceptible to $S$. sclerotiorum, with the largest lesions. To our knowledge, this is the first report of high levels of resistance to $S$. sclerotiorum in B. oleracea at the cotyledon stage and also the first report of the host cotyledon reactions against $S$. sclero-
\end{abstract}

tiorum for all tested species except $B$. napus and $B$. juncea. The mean lesion size for $B$. napus introgressed with $B$. carinata was $5.6 \mathrm{~mm}$, which is midway between the lesion size for the two parent species $B$. napus $(5.1 \mathrm{~mm})$ and B. carinata $(5.8 \mathrm{~mm})$. Separate genetic control for cotyledon versus mature plant resistance was demonstrated by the lack of correlation between lesion size from $S$. sclerotiorum on the cotyledon with the severity of disease initiated by stem inoculation or natural processes in a previous field test. On the most resistant genotypes, B. oleracea var. italica Prophet and var. capitata Burton, growth of $S$. sclerotiorum on the cotyledon surface prior to penetration was severely impeded, production of appressoria inhibited, and both cytoplasm shrinkage and protoplast extrusion in $S$. sclerotiorum hyphae prevalent. This is the first report of such resistant mechanisms in $B$. oleracea. Genotypes with cotyledon resistance identified in this study will be of great value not only in furthering our understanding of resistance mechanisms across different cruciferous species but also could be exploited for developing commercial crucifer cultivars with highlevel resistance against $S$. sclerotiorum.
The family Brassicaceae contains important crop species used for broadacre oilseed, forage, and vegetable production. Species used for oilseed production include oilseed rape (Brassica napus and $B$. rapa) and oilseed mustards such as Indian mustard ( $B$. juncea), Ethiopian mustard (B. carinata), and black mustard ( $B$. nigra) (35). Important broadacre dual-purpose or straight forageproduction species include oilseed rape (B. napus) and kale (B. oleracea) (2), while many variants of $B$. oleracea are used for vegetable production, along with Asian brassicas (e.g., B. rapa var. rosularis) and other species such as Raphanus spp., which can be important crops regionally (34). B. napus, B. oleracea, and Raphanus spp. can all be included in "mustard and cress" seedling mixtures grown for consumption as salad vegetable mixes (18).

Sclerotinia sclerotiorum causes serious endemic disease across oilseed brassicas, forage brassicas, and vegetable brassicas $(2,21,36)$. S. sclerotiorum also causes damping off in a variety of plants, causing losses at the seedling stage and significantly affecting transplant production (23). This pathogen has a wide host range (5) and survives in soil in the absence of a host for several years as thick-walled resting sclerotia (1). S. sclerotiorum attacks plants in a variety of ways in the field. Sclerotia can germinate carpogenically, releasing ascospores that infect petals, leaves, and

Corresponding author: M. J. Barbetti, E-mail: martin.barbetti@uwa.edu.au

Accepted for publication 26 August 2013.

http://dx.doi.org/10.1094/PDIS-06-13-0612-RE

(C) 2014 The American Phytopathological Society stems. It is generally accepted that the most common route for stem infection of oilseed brassicas is through the infection of petals, which then fall and adhere to the stem, providing a nutritional resource that allows $S$. sclerotiorum to penetrate and infect the stem (17). However, direct infection of leaves and stems by ascospores in the absence of flower petals also seems possible (38) and sclerotia can germinate myceliogenically, entering the stem at ground level, as commonly occurs on mustard crops (B. juncea) in India (36).

Currently, cultural and chemical controls provide only partial control and can be cost prohibitive, particularly for broadacre oilseed Brassica crops in countries such as Australia (3), and locating effective resistance to $S$. sclerotiorum remains a high priority across cruciferous crops worldwide. Resistance that reduces seedling losses would be valuable for improving crop establishment and survival and could be vitally important where Brassica seedlings are grown for consumption as salad, such as in "mustard and cress" mixtures. There have been recent successes in locating high levels of host resistance to the stem rot phase in oilseed rape and mustards, especially in Brassica genotypes following hybridization of B. napus or B. juncea with three wild crucifers (viz., Erucastrum cardaminoides, Diplotaxis tenuisiliqua, and E. abyssinicum $)(11,26,27)$ and the testing of a diverse group of crucifers revealed very high levels of resistance to Sclerotinia stem rot (SSR) in selections of Raphanus spp., B. carinata, and B. napus (38).

Various methods have been developed for testing resistance to $S$. sclerotiorum in brassicas. Field evaluations of SSR, while generally considered the most appropriate and reliable technique for evaluating stem resistance against SSR, can still result in highly 
variable findings, particularly where fluctuating environmental conditions influence the response of individual genotypes $(27,31)$. For this reason, controlled-environment screening methods have been widely used to assess physiological resistance to $S$. sclerotiorum in various crops. A cotyledon test has been developed for screening B. napus genotypes against $S$. sclerotiorum at the seedling stage (15) but its potential application across more diverse cruciferous species has not been evaluated. In addition, although the seedling cotyledon test on B. napus showed a significant positive correlation $(r=0.62)$ between SSR test results in the assay and in the field (15), some studies have suggested that different mechanisms govern the resistance of brassicas to $S$. sclerotiorum at different growth stages. For example, Zhao et al. (39) detected six quantitative trait loci associated with resistance to $S$. sclerotiorum in oilseed rape (B. napus), three of which were associated with cotyledon resistance at the seedling stage and the remaining three with stem resistance at the maturing stage. Our understanding of the expression of resistance to $S$. sclerotiorum could be increased by further comparisons between SSR at seedling and adult host stages, including observation of the growth of the fungus on the host.

The aim of this study was to define the range and extent of seedling resistance to $S$. sclerotiorum across a variety of oilseed, for- age, and vegetable crucifers, including wild or weedy species, and $B$. napus containing B genome introgressions from $B$. carinata. In addition, we aimed to determine the correlation between resistance found at the seedling stage with a previous field assessment (38) and observe the growth of the pathogen on susceptible and resistant cotyledons.

\section{Materials and Methods}

Fungal isolate. $S$. sclerotiorum isolate MBRS-1, collected from infected tissue of B. napus in the Mount Barker region of Western Australia in 2004 (26), was used. Isolate MBRS-1 was chosen because it is highly pathogenic (12) and belongs to the prevailing pathotype (pathotype 76) occurring in Western Australia (16). This isolate has been extensively used for resistance screening of $B$. juncea and $B$. napus genotypes in both the glasshouse and the field $(11,15,16,25-27)$.

Genotypes tested. In total, 46 cruciferous genotypes from 12 different species were tested (Table 1). The majority of genotypes tested were obtained from a seed collection held at the University of Western Australia, supplied by Dr. Bob Redden at the Australian Temperate Field Crops Collection at Department of Primary Industries, Horsham, Victoria, Australia, or purchased from commercial

Table 1. Average cotyledon width and lesion diameter on the cotyledons of 46 cruciferous genotypes $48 \mathrm{~h}$ after inoculation with Sclerotinia sclerotiorum

\begin{tabular}{|c|c|c|c|c|}
\hline Genotype name & Species & Origin & Lesion diameter $(\mathrm{mm})^{\mathrm{a}}$ & Cotyledon width $(\mathrm{mm})$ \\
\hline Prophet & Brassica oleracea var. italica & sAustralia & $0.8(1)$ & 15.4 \\
\hline Burton & B. oleracea var. capitata & sAustralia & $2.3(2)$ & 15.3 \\
\hline Beverly Hills & B. oleracea var. capitata & sAustralia & $3.6(3)$ & 12.3 \\
\hline BTO 2 & B. tournefortii & Australia & $4.0(4)$ & 7.8 \\
\hline Choy sum & B. rapa var. chinensis & sAustralia & $4.2(5)$ & 9.9 \\
\hline SAR22 & Sinapis arvensis & Australia & $4.3(6)$ & 7.7 \\
\hline YM16 & B. napus & China & $4.4(7)$ & 16.4 \\
\hline 94697 & B. incana & sAustralia & $4.5(8)$ & 10.5 \\
\hline 06-P71-2 & B. napus & China & $4.6(9)$ & 16.3 \\
\hline Tatsoi & B. rapa var. rosularis & sAustralia & $4.7(10)$ & 8.9 \\
\hline ZY005 & B. napus & China & $4.7(11)$ & 9.9 \\
\hline YM04 & B. napus & China & $4.7(12)$ & 15.5 \\
\hline BTO 6 & B. tournefortii & Israel & $4.8(13)$ & 8.3 \\
\hline SAR 5 & S. arvensis & Australia & $4.8(14)$ & 9.9 \\
\hline ZY004 & B. napus & China & $4.8(15)$ & 15.1 \\
\hline Charlton & B. napus & Australia & $4.9(16)$ & 11.0 \\
\hline NC2-6 & B. napus with $B$. carinata introgression & India & $5.0(17)$ & 16.0 \\
\hline PI 193459 & B. carinata & Ethiopia & $5.1(18)$ & 12.5 \\
\hline BRA $926 / 78$ & B. carinata & Ethiopia & $5.1(19)$ & 15.5 \\
\hline NC5 & B. napus with $B$. carinata introgression & India & $5.1(20)$ & 14.5 \\
\hline BRA 926/81 & B. carinata & Ethiopia & $5.3(21)$ & 15.4 \\
\hline YM11 & B. napus & China & $5.3(22)$ & 18.5 \\
\hline Boss & Raphanus sativus & sAustralia & $5.4(23)$ & 15.5 \\
\hline YM13 & B. napus & China & $5.4(24)$ & 16.9 \\
\hline Black mustard & B. nigra & sAustralia & $5.4(25)$ & 10.9 \\
\hline 054103 & B. carinata & Ethiopia & $5.4(26)$ & 16.8 \\
\hline JO006 & B. juncea & Australia & $5.4(27)$ & 11.7 \\
\hline 054107 & B. carinata & Ethiopia & $5.5(28)$ & 15.0 \\
\hline YM07 & B. napus & China & $5.5(29)$ & 17.6 \\
\hline UPM6563 & B. incana & sAustralia & $5.6(30)$ & 12.9 \\
\hline JM06006 & B. juncea & Australia & $5.6(31)$ & 11.3 \\
\hline 054106 & B. carinata & Ethiopia & $5.7(32)$ & 15.6 \\
\hline Mystic & B. napus & Australia & $5.7(33)$ & 16.0 \\
\hline NC9 & B. napus with $B$. carinata introgression & India & $5.8(34)$ & 16.3 \\
\hline WARR 7 & R. raphanistrum & Australia & $5.8(35)$ & 12.4 \\
\hline BCA 1 & B. carinata & Australia & $5.8(36)$ & 17.3 \\
\hline $\mathrm{NC} 11-2$ & B. napus with $B$. carinata introgression & India & $6.0(37)$ & 17.4 \\
\hline NC3-4 & B. napus with $B$. carinata introgression & India & $6.0(38)$ & 16.9 \\
\hline 054113 & B. carinata & Ethiopia & $6.1(39)$ & 15.0 \\
\hline YM10 & B. napus & China & $6.2(40)$ & 15.6 \\
\hline JM06018 & B. juncea & Australia & $6.3(41)$ & 12.8 \\
\hline 054104 & B. carinata & Ethiopia & $6.4(42)$ & 16.9 \\
\hline WARR 20 & R. raphanistrum & Australia & $6.4(43)$ & 15.3 \\
\hline SAR 7 & S. arvensis & Australia & $6.6(44)$ & 11.0 \\
\hline Xinyou 9 & B. juncea & China & $6.9(45)$ & 14.8 \\
\hline SMP3-82 & B. carinata & Pakistan & $7.3(46)$ & 14.8 \\
\hline
\end{tabular}

${ }^{\mathrm{a}}$ Lesion diameter was analyzed using analysis of variance $(P=0.001)$ and means were separated using Fisher's least significant difference $(5 \%)=1.14$. Relative genotype ranking for lesion diameter is shown in parentheses, where 1 represents the most resistant genotype and 46 represents the most susceptible genotype. sAustralia indicates that seed was sourced in Australia. 
seed companies. B. oleracea 'Prophet' was obtained from Fairbanks Seeds, 'Burton' and 'Beverley Hills' from Lefroy Valley Seeds, and B. rapa 'Choy sum' and 'Tatsoi' from Johnson's Seeds. The test genotypes were selected on the basis of their diversity, as well as performance in earlier field or cotyledon tests $(15,38)$.

Cotyledon inoculation experiment. Plants were grown in a pasteurized compost mixture in 35-by-29-by-6-cm trays, each having 30 individual cells. Five seeds of each genotype were sown in each cell and thinned to two seedlings per cell after emergence. Groups of two trays, together containing all 46 test genotypes, were placed in separate 30 -liter clear plastic storage boxes with lids. The trays were incubated in a controlled environment growth room, set to $18 \pm 1{ }^{\circ} \mathrm{C}$ during the day and $14 \pm 1^{\circ} \mathrm{C}$ at night, on a 12-h light-dark cycle, with light intensity of $220 \mu \mathrm{M} \mathrm{m}^{-2} \mathrm{~s}^{-1}$. Seedlings were grown until their cotyledons were fully expanded, equivalent to growth stage 1.00 on the Sylvester-Bradley and Makepeace scale (37).

Production of inoculum and inoculations were based on the methods of Garg et al. (15). Five discs were excised from the actively growing margin of 3-day-old colonies of $S$. sclerotiorum grown on potato dextrose agar at $20^{\circ} \mathrm{C}$ and used to inoculate 150 $\mathrm{ml}$ of liquid growth medium ( $24 \mathrm{~g}$ of potato dextrose broth, $10 \mathrm{~g}$ of peptone, and 1 liter of $\mathrm{H}_{2} \mathrm{O}$ ) in 250-ml flasks. The cultures were shaken at $150 \mathrm{rpm}$ at $20^{\circ} \mathrm{C}$. After 3 days, actively growing mycelium was harvested by straining the broth through four layers of cheesecloth and washing with deionized water. The fungal mats obtained were then transferred to $125 \mathrm{ml}$ of the same liquid medium and macerated using a hand-held blender for $3 \mathrm{~min}$. The concentration of mycelia in the suspension was measured using a hemocytometer (Superior) and adjusted to $1 \times 10^{4}$ fragments $/ \mathrm{ml}$ by diluting with fresh growth medium.

Cotyledon width (two lobes) was measured with a ruler immediately prior to inoculation. A single 10- $\mu$ d droplet of mycelial suspension was spotted onto each of the four lobes of each seedling using a micropipette. The inoculum was shaken often to maintain a homogeneous mycelial suspension. It was noted that the initial droplet of inoculum spread to cover an area approximately $3 \mathrm{~mm}$ in diameter. Where a genotype had a small leaf size $(<10 \mathrm{~mm})$, only two droplets were applied to each plant (i.e., one droplet per cotyledon), and double the number of plants were inoculated to compensate.

Plants were saturated on the morning of their inoculation and a high-humidity environment attained by spraying a fine mist of water on the inside of the containers before closing the lids and placing the boxes in sealed, black plastic bags. Lesion diameter was measured in millimeters $48 \mathrm{~h}$ post inoculation (hpi), as described by Garg et al. (15), who presented this technique as a reliable means of comparing the level of resistance in B. napus genotypes. A complete randomized block design was used with four replications and eight measurements (four droplets on each of two seedlings) per replication. The entire experiment was conducted twice. Lesion diameter was analyzed separately for the two experiments using analysis of variance with Genstat (14th edition, Lawes Agricultural Trust). Fisher's least significant differences $(P<0.05)$ were used to compare genotype reactions. The relationship between the two experiments was assessed using a one-sided paired $t$ test in Genstat. The paired $t$ test detected no difference between experiments $(P>0.05)$; therefore, all data were consolidated and reanalyzed together. Regression analyses were undertaken using the regression function in Microsoft Excel to examine the relationship between the lesion diameters on cotyledons in this study and the length of stem lesions after inoculation with $S$. sclerotiorum MBRS-1 and incidence of natural leaf infection by $S$. sclerotiorum on the same genotypes in an associated field trial (38).

Anatomical studies on the infection process of $S$. sclerotiorum. Growth of MBRS-1 on cotyledon tissues of two genotypes previously determined to be resistant to $S$. sclerotiorum (B. oleracea var. italic Prophet and B. oleracea var. capitata Burton) and three genotypes found to be susceptible to S. sclerotiorum (B. carinata 054104 , B. carinata 054106 , and Raphanus raphanistrum WARR 20) were sampled at $24 \mathrm{hpi}$ and decolorized in acetic acid/ethanol/water $(2: 2: 1)$ for 5 days at $25^{\circ} \mathrm{C}$, with the solution replaced with fresh where required to complete the decolorization. At least four cotyledons of each genotype were examined in two repeated experiments. At the time of examination, cotyledons were washed with two changes of deionized water and stained with $0.05 \%$ (wt/vol) aniline blue for $90 \mathrm{~s}$. Stained cotyledons were wet mounted whole on glass microscope slides, examined, and photographed (Olympus BX51 microscope and DP71 digital photographic system for bright field optics, or Olympus SZX10 stereo microscope and DP21 digital photographic system). After examination of the stained cotyledons, an attempt was made to remove the hyphal mat with tweezers, to determine how firmly it adhered to the surface of the cotyledon. Several seedlings from each genotype were observed 48 hpi to ensure that disease progress was consistent with the earlier experiments.

\section{Results}

Diameter of lesions caused by $\boldsymbol{S}$. sclerotiorum on cruciferous genotypes. Lesion diameters following inoculation with MBRS-1 varied significantly $(P<0.001)$ among the cruciferous genotypes (Table 1). There was a significant relationship between the width of a genotype's cotyledons and the diameter of lesions resulting from S. sclerotiorum infection on that genotype $(P<0.004, r=$ $0.41)$. However, when the two genotypes with the largest cotyledons, $R$. sativus Krasnodar Market B (average cotyledon width = $22.6 \mathrm{~mm}$, average lesion width $=8.51 \mathrm{~mm}$ ) and $R$. sativus $\mathrm{Mi}$ nowase $\mathrm{Nr} 2$ (average cotyledon width $=23.5 \mathrm{~mm}$, average lesion width $=7.5 \mathrm{~mm}$ ) were excluded from the analysis, this relationship between cotyledon width and lesion diameter disappeared $(P=$ $0.14, r=0.22)$. R. sativus Krasnodar Market B and Minowase $\mathrm{Nr} 2$ were removed from all subsequent analyses, and the mean lesion diameter at 48 hpi for the remaining genotypes ranged from 0.8 $\mathrm{mm}$ for B. oleracea var. italica Prophet to $7.3 \mathrm{~mm}$ for $B$. carinata SMP3-82 (Table 1).

When lesion diameters on cotyledons under controlled environmental conditions in this study were compared with the length of stem lesions caused by $S$. sclerotiorum in the field in a previous study (38), there was no significant relationship between them $(P=$ $0.42, r=0.14)$. Likewise, there was no significant correlation between lesion size on cotyledons and the incidence of natural leaf infection in the field $(P=0.21, r=0.22)$. In fact, the only selections with a reaction that was congruent between the cotyledon test and the stem test were $B$. juncea 'Xinyou 9' and $B$. carinata 'SMP3-82', which were highly susceptible in both studies. Of the other genotypes, B. oleracea var. italica Prophet was the most resistant in the cotyledon assay (mean lesion diameter $=0.8 \mathrm{~mm}$ ) but was midway in genotype rankings for SSR in the field. Similarly, B. rapa var. chinensis Choy sum was highly susceptible to SSR in the field but ranked fifth most resistant in the cotyledon test (mean lesion width $=4.2 \mathrm{~mm}$ ). Examples of genotypes that displayed resistance in the field but were highly susceptible in the cotyledon test included B. carinata 054104 (mean lesion diameter $=6.4 \mathrm{~mm}), B$. carinata 054113 (mean lesion diameter $=6.1 \mathrm{~mm})$, $B$. napus 'Mystic' (mean lesion diameter $=5.7 \mathrm{~mm}$ ), and $R$. raphanistrum WARR 20 (mean lesion diameter $=6.4 \mathrm{~mm}$ ). B. napus 'Charlton' and Mystic were both in the midrange for mean lesion diameter in this experiment, in spite of the fact that Mystic was highly resistant in a previous cotyledon evaluation (15) and had the smallest stem lesions in the field trial.

Variation in lesion diameter caused by $\boldsymbol{S}$. sclerotiorum within a species. There was apparent variation between different genotypes within a single species in terms of lesion diameter (Table 2). The two species with the largest number of representatives $(B$. carinata and $B$. napus) did not have the widest range in lesion width. In fact, $B$. oleracea was the species with the largest variation, in spite of being represented by only three genotypes. The five selections of $B$. napus introgressed with $B$. carinata were close to one another in terms of cotyledon lesion size from $S$. scleroti- 
orum, with a maximum range of only $0.9 \mathrm{~mm}$. The mean lesion size for these latter five selections $(5.6 \mathrm{~mm})$ was midway between the lesions observed on the two parent species, B. napus $(5.1 \mathrm{~mm})$ and B. carinata $(5.8 \mathrm{~mm})$.

Infection process of $\boldsymbol{S}$. sclerotiorum. In all genotypes, the droplets of inoculum applied to the cotyledon surface initially remained discrete from the leaf surface, with surface tension maintaining a rounded shape. In all instances, the mycelial fragments of S. sclerotiorum grew using the nutrients in the droplet, forming a hyphal mat on the cotyledon surface. In most cases, by 24 hpi, hyphae had extended beyond the limits of the droplet and appressoria were forming at the edge of the hyphal mat (Fig. 1A and B).

In $B$. oleracea var. italica Prophet at $24 \mathrm{hpi}$, the hyphal mat was confined to the area of the inoculation droplet and no appressoria were formed (Fig. 1C). If the hyphal mat was removed at $24 \mathrm{hpi}$, there was little macroscopic evidence of the inoculation having ever occurred, except for small necrotic flecks on the surface of the cotyledon in the area of the inoculation droplet, indicative of a hypersensitive response (HR). The hyphae present on the cotyledon showed cytoplasmic shrinkage and protoplastic extrusion and, with the latter symptom, extensively diffuse cytoplasmic contents were evident around the hyphae (Fig. 1D).

Macroscopically, the initial site of infection on the cotyledons was frequently at the edge of the hyphal mat, often giving the appearance of "pitting" (Fig. 1E). In most cases, the infection was so advanced by 48 hpi that the extensive hyphal growth in the lesion had destroyed the structure of this section of the cotyledon. Because the majority of seedlings were very susceptible to attack by S. sclerotiorum, the test was concluded at $48 \mathrm{hpi}$, prior to the more susceptible seedlings being completely consumed by the pathogen. In B. oleracea var. italica Prophet at 48 hpi, however, almost no $S$. sclerotiorum hyphae were observed on any part of the cotyledon and the infection process had ceased, while discoloration and spotting typical of an HR was evident on the cotyledon surface (Fig. $1 \mathrm{~F})$. Hyphae growing on $B$. oleracea var. capitata Burton also showed clear evidence of both cytoplasmic shrinkage and protoplastic extrusion (Fig. 1G) but, by 48 hpi, more substantial lesions were visible on the cotyledons than the HR observed with $B$. oleracea var. italica Prophet.

Browning of epidermal and upper cortical tissues was sometimes observed at high magnification directly underneath appressoria by $24 \mathrm{hpi}$; for example, on $R$. raphanistrum WARR 20 (Fig. $1 \mathrm{H})$ and $B$. carinata 054106 . However, for both of these genotypes, no HR was observed on the inoculated cotyledons and no shrinkage of cytoplasm away from cell walls or protoplast extrusion from hyphae was observed.

\section{Discussion}

These experiments, involving diverse cruciferous genotypes, including genotypes carrying introgression from $B$. carinata, highlight the enormous potential for incorporating high levels of seedling resistance to $S$. sclerotiorum into commercial germplasm of cruciferous oilseed, forage, and vegetable crops. This is the first time that cotyledon seedling reactions to $S$. sclerotiorum have been reported on $B$. carinata, $B$. incana, $B$. napus introgressed with $B$. carinata, B. nigra, B. oleracea, B. rapa var. rosularis, B. rapa var. chinensis, B. tournefortii, $R$. raphanistrum, $R$. sativus, and Sinapis arvensis.

We observed a high level of resistance to Sclerotinia sclerotiorum in the cotyledons of B. oleracea, compared with partial resistance reported in other tissues of B. oleracea and its wild relatives $(8,10,32)$. Further, the black "flecking" on the surface of the cotyledon in the area of the inoculation droplet indicated an HR on $B$. oleracea var. italica Prophet, similar to that previously reported in B. napus Charlton challenged with S. sclerotiorum (14). We believe this to be the first report of an HR to $S$. sclerotiorum on cotyledons of $B$. oleracea. Cytoplasmic shrinkage and protoplastic extrusion for $S$. sclerotiorum hyphal cells, as observed on both $B$. oleracea var. italica Prophet and var. capitata Burton, were previously reported on crucifer seedling cotyledons for B. napus Charl- ton (14). It is noteworthy that, in our study, we readily observed these symptoms at 1 day post inoculation (dpi) compared with 4 dpi. Our study is the first protoplast extrusion from hyphal cells reported in the $B$. oleracea-S. sclerotiorum pathosystem. A similar phenomenon was also observed in B. napus by Garg et al. (14). We concluded that the contents emerging from the end or at the center of the fungal hyphae were protoplast extrusions and not appressoria because of the extensive shrinkage of the cytoplasmic content of the hyphal cells, while appressoria of S. sclerotiorum on the surface of a susceptible host genotype always arose from dichotomous branching of the terminal hyphae (30), not from the intercalary hyphal cells, as we observed here. The occurrence of protoplast extrusion from hyphal cells, and only on the resistant genotypes, points to the possibility of an as-yet-undefined defense mechanism in B. oleracea and suggests that it may be similar to that found in B. napus Charlton $(13,14)$.

It is noteworthy that the inhibition of $S$. sclerotiorum infection by $B$. oleracea var. italica Prophet occurred prior to any obvious visual damage to the host. Initial growth of S. sclerotiorum in a hyphal mat was evident prior to infection on both resistant and susceptible selections and protoplasmic extrusion was observed in this phase on B. oleracea var. italica Prophet. Together, these results strongly suggest the production of antifungal compounds by the host in response to infection. S. sclerotiorum is known to produce elicitors such as oxalic acid and a variety of polygalacturonases that move into host tissue ahead of its infection hyphae $(6,29)$. These or similar compounds produced in the hyphal mat may have stimulated an active defense by $B$. oleracea var. italica Prophet. A complex mixture of defense metabolites with antifungal activity are produced by crucifers when they are attacked by pathogens, including phytoalexins such as brassilexin and sinalexin (33). Further work to fully understand the mechanism by which $S$. sclerotiorum is destroyed on the surface of resistant cotyledons is clearly warranted.

Why cotyledons of B. napus Charlton and Mystic were not highly resistant to $S$. sclerotiorum in our experiments, in contrast to their previous performance in a similar test, remains unexplained; however, it is possible that the use of a different seed lot was responsible (14). Seed source is known to affect expression of resistance to other diseases in brassicas, such as blackleg (Leptosphaeria maculans; M. J. Barbetti, unpublished data). The intermediate status of cotyledons between embryo and leaf (7) may mean that the metabolic fitness of the seed impacts the ability of the cotyledons to respond to challenge by a pathogen. Both $B$. oleracea var. italica Prophet and var. capitata Burton were F1 hybrid seed obtained fresh from commercial seed sources, whereas seed for many of the other selections, including B. napus Charlton and Mystic, had been stored for varying lengths of time and up to 8 years at $4^{\circ} \mathrm{C}$. Further examination of the effect of seed age and

Table 2. Number of genotypes tested, average lesion width and range in lesion widths caused by Sclerotinia sclerotiorum for each species

\begin{tabular}{lccc}
\hline Species & $\boldsymbol{N}^{\mathbf{a}}$ & $\begin{array}{c}\text { Lesion } \\
(\mathbf{m m})^{\mathbf{b}}\end{array}$ & $\begin{array}{c}\text { Range } \\
(\mathbf{m m})^{\mathbf{c}}\end{array}$ \\
\hline Brassica rapa var. chinensis & 1 & 4.2 & $\ldots$ \\
B. rapa var. rosularis & 1 & 4.7 & $\ldots$ \\
Raphanus sativus & 1 & 5.4 & $\ldots$ \\
B. nigra & 1 & 5.4 & $\ldots$ \\
B. tournefortii & 2 & 4.4 & 0.8 \\
B. incana & 2 & 5.0 & 1.1 \\
R. raphanistrum & 2 & 6.1 & 0.6 \\
B. oleracea & 3 & 2.2 & 2.7 \\
Sinapis arvensis & 3 & 5.3 & 2.3 \\
B. juncea & 4 & 6.1 & 1.4 \\
B. napus with B. carinata introgression & 5 & 5.6 & 1.0 \\
B. carinata & 10 & 5.8 & 2.1 \\
B. napus & 11 & 5.1 & 1.8 \\
\hline
\end{tabular}

a Number of genotypes tested.

b Average lesion diameter.

c Range of variation in lesion diameter. 
production conditions on the expression of cotyledon resistance to S. sclerotiorum would be useful.

There appeared to be greater uniformity in reaction to $S$. sclerotiorum within representatives of a particular species in this experiment testing reactions on cotyledons than occurred in field trials for assessing resistance to SSR (38). This may be a consequence, in part, of the much tighter control over environmental conditions for the cotyledon screening studies compared with the stem infection studies under more variable field conditions, par- ticularly because expression of intermediate levels of host resistance is particularly influenced by changes in environmental conditions (20).

Inoculating with droplets of fungal mycelium in potato dextrose broth containing peptone mimicked the natural infection processes. The growth of the mycelium through the droplet and formation of a hyphal mat prior to infection of the leaf mirrors the natural process of S. sclerotiorum, which uses infected petals as a source of nutrition prior to penetration of the leaf or stem (17). However, there
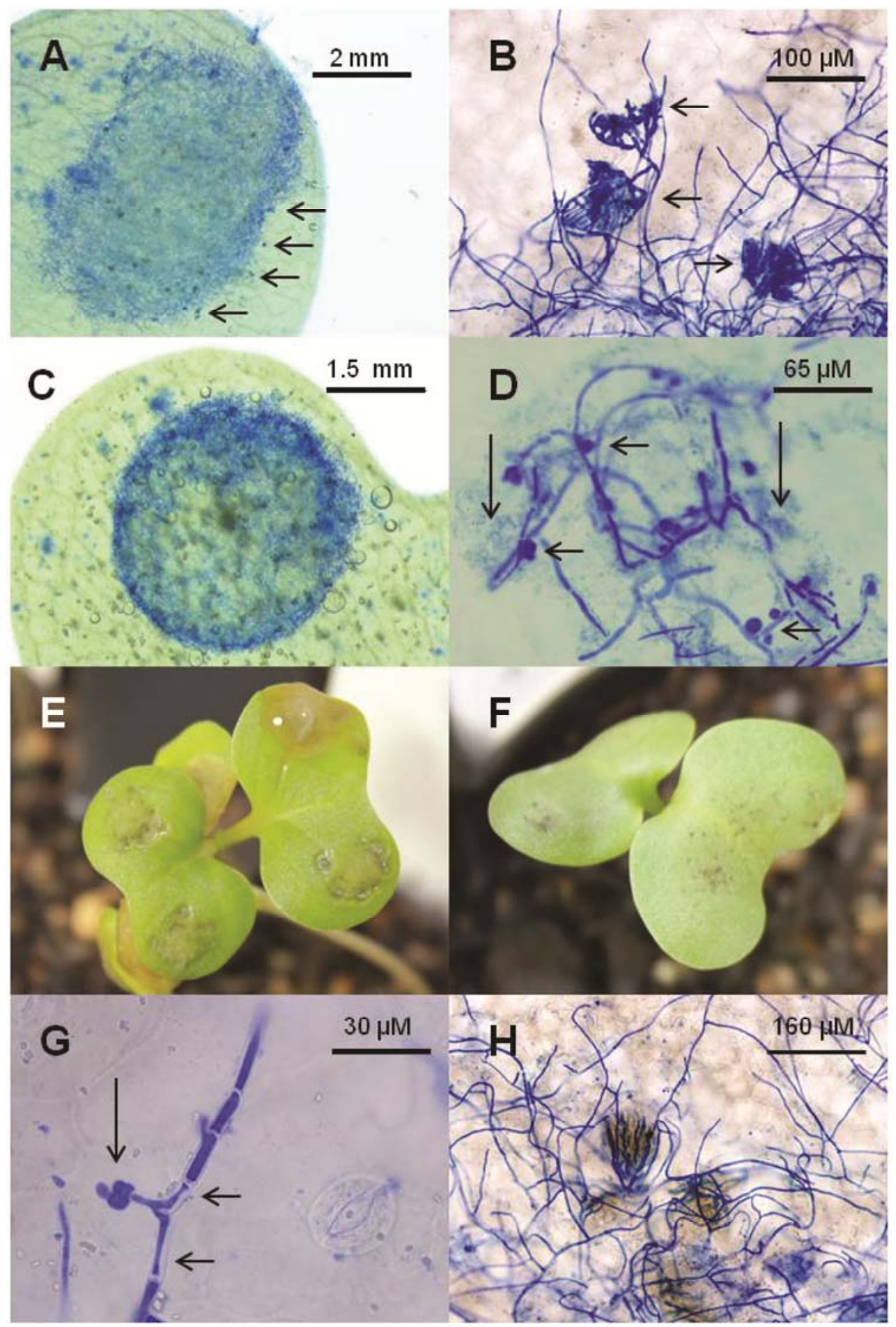

Fig. 1. A, Brassica carinata 054106 with hyphal mat at $24 \mathrm{~h}$ post inoculation (hpi). Appressoria are visible at the edge of the lesion (arrows). B, Higher magnification of A showing appressoria at edge of hyphal mat in B. carinata 054106 at $24 \mathrm{hpi}$, with repeated dichotomous branching of the terminal hyphae (arrows) leading to the formation of appressoria (arrows). C, B. oleracea var. italica 'Prophet' with hyphal mat at $24 \mathrm{hpi}$. D, Higher magnification of C showing B. oleracea var. italica Prophet with protoplast extrusion (longer arrows) and diffused cytoplasmic contents surrounding affected mycelium (shorter arrows). E, Example of infection starting from the edge of the hyphal mat on B. napus introgressed with B. carinata NC11-2 at 48 hpi. F, Typical appearance of B. oleracea var. italica Prophet at 48 hpi. G, B. oleracea 'Burton', ×100 magnification showing typical symptoms of cytoplasm shrinkage (shorter arrows) and cytoplasmic extrusion (longer arrows). H, Brown discoloration observed on Raphanus raphanistrum WARR 20 directly underneath the location of appressoria at $24 \mathrm{hpi}$. 
was a lack of correlation between the results of tests for a given genotype at the cotyledon stage in this study with either adult plant stem resistance following artificial inoculation or naturally occurring leaf infection across a similar diverse range of cruciferous genotypes in field studies (38). Differences in the expression of resistance in juvenile and adult plants has already been reported in brassicas for other disease-host combinations such as L. maculans in oilseed rape (B. napus) (28), downy mildew (Hyaloperonospora parasitica) in broccoli (9), and Cauliflower mosaic virus and Pseudomonas syringae in Arabidopsis (22,24). More specifically, in reactions to $S$. sclerotiorum, several studies have indicated that there are differences between juvenile and adult plant reactions in B. napus $(4,19)$ and that the mechanisms of resistance are different in brassicas of different ages (39). The only genotypes in our study with reactions which were congruent between adult stem and cotyledon tests were the two most susceptible selections, B. juncea Xinyou 9 and $B$. carinata SMP3-82. Kim et al. (20), looking at three different methods of assessing the resistance of soybean cultivars to $S$. sclerotiorum, also found that highly susceptible genotypes were consistent between tests, whereas cultivars with intermediate reactions were not. This topic needs further investigation in relation to cotyledon seedling resistance in crucifers. It is now evident that, outside of B. napus, field resistance cannot be predicted from cotyledon resistance and should be tested separately to locate the seedling or adult plant resistance needed.

Preliminary tests in our study soon revealed the challenges in expanding the use of the technique proposed by Garg et al. (15) as a method of assessing the susceptibility of a variety of genotypes of $B$. napus to be across a more diverse range of cruciferous species. For example, it was evident that cotyledons of Sisymbrium irio, D. tenuifolia, and Camelina sativa had lobes smaller than the diameter of the inoculation droplet applied to B. napus (approximately 3 $\mathrm{mm}$ in diameter); therefore, crucifers with an average cotyledon width less than $7 \mathrm{~mm}$ were excluded from our study. Where cotyledon width is between 7 and $10 \mathrm{~mm}$, an adjustment can be made by applying the inoculum to only one lobe instead of two, enabling realistic comparisons between small-lobed genotypes and genotypes with larger cotyledons. Genotypes with very large cotyledons were also excluded to remove a slight positive correlation between cotyledon size and lesion size where genotypes with cotyledons greater than $22 \mathrm{~mm}$ were included. Although the need to exclude genotypes with cotyledons less than $7 \mathrm{~mm}$ or greater than $22 \mathrm{~mm}$ restricts the application of this technique to assessing the physiological susceptibility of some crucifer species to $S$. sclerotiorum at the cotyledon stage, there was excellent differentiation between the reactions to $S$. sclerotiorum across species as varied as $R$. raphanistrum, B. carinata, B. oleracea, and $S$. arvensis. Thus, the expansion of the cotyledon screening technique to a wider range of new genera and species was successful and provides the first new information on cotyledon reactions against $S$. sclerotiorum for these previously untested species.

Because current management of S. sclerotiorum is often suboptimal (3), there is an urgency to develop and commercially utilize new host resistances such as those identified in this study. To our knowledge, this is the first report of high levels of resistance in $B$. oleracea at the cotyledon stage. The results also provide evidence for a disparity in resistance reactions between juvenile and mature plants of the same host genotype. Although most studies of seedling resistance to $S$. sclerotiorum seem to have been undertaken with the hope of developing a quick and efficient estimate of adult plant resistance, the inherent value of resistance at the seedling stage should not to be ignored because it would improve crop establishment and survival. It is vital that assessment of resistance to S. sclerotiorum should be undertaken at all important stages of plant growth to ensure commercial relevance.

\section{Acknowledgments}

Financial assistance to $M$. Uloth was provided through an Australian Postgraduate Award. We thank the Australia Research Council and the Depart- ment of Agriculture and Food Western Australia for providing operational funding support for this research; B. Redden, Curator, Australian Temperate Field Crops Collection, Horsham, Victoria for supplying seed lines with the ATC prefix; and B. He for excellent assistance with the microscopy components of this study.

\section{Literature Cited}

1. Adams, P. B., and Ayers, W. A. 1979. Ecology of Sclerotinia species. Phytopathology 69:896-899.

2. Ayres, L., and Clements, B. 2002. Forage brassicas-quality crops for livestock production. AgFact P2.1.13. NSW Agriculture, Orange, Australia

3. Barbetti, M. J., Li, C. X., Garg, H., Li, H., Banga, S. K., Banga, S. S., Sandhu, P. S., Singh, R., Singh, D., Liu, S. Y., Gurung, A. M., and Salisbury, P. A. 2011. Host resistance in oilseed Brassicas against Sclerotinia-renewed hope for managing a recalcitrant pathogen. Pages 713-715 in: Proc. 13th Int. Rapeseed Congr. Prague, Czech Republic.

4. Behla, R. S. 2011. Identification of quantitative trait loci for resistance to Sclerotinia sclerotiorum in Brassica napus. Ph.D. thesis, University of Manitoba, Winnipeg, Canada.

5. Boland, G. J., and Hall, R. 1994. Index of plant hosts of Sclerotinia sclerotiorum. Can. J. Plant Pathol. 16:93-108.

6. Cessna, S. G., Sears, V. E., Dickman, M. B., and Low, P. S. 2000. Oxalic acid, a pathogenicity factor for Sclerotinia sclerotiorum, suppresses the oxidative burst of the host plant. Plant Cell 12:2191-2200.

7. Chandler, J. W. 2008. Cotyledon organogenesis. J. Exp. Bot. 59:2917-2931.

8. Cubeta, M. A., Cody, B. R., Kohli, Y., and Kohn, L. M. 1997. Clonality in Sclerotinia sclerotiorum on infected cabbage in Eastern North Carolina. Phytopathology 87:1000-1004.

9. Dickson, M. H., and Petzoldt, R. 1993. Plant age and isolate source affect expression of downy mildew resistance in broccoli. Hortscience 28:730731.

10. Dickson, M. H., and Petzoldt, R. 1996. Breeding for resistance to Sclerotinia sclerotiorum in Brassica oleracea. Acta Hortic. 407:103-108.

11. Garg, H., Atri, C., Sandhu, P. S., Kaur, B., Renton, M., Banga, S. K., Singh, H., Singh, C., Barbetti, M. J., and Banga, S. S. 2010. High level of resistance to Sclerotinia sclerotiorum in introgression lines derived from hybridization between wild crucifers and the crop Brassica species B. napus and B. juncea. Field Crops Res. 117:51-58.

12. Garg, H., Kohn, L. M., Andrew, M., Li, H., Sivasithamparam, K., and Barbetti, M. J. 2010. Pathogenicity of morphologically different isolates of Sclerotinia sclerotiorum with Brassica napus and B. juncea genotypes. Eur. J. Plant Pathol. 126:305-315.

13. Garg, H., Li, H., Sivasithamparam, K., and Barbetti, M. J. 2013. Differentially expressed proteins and associated histological and disease progression changes in cotyledon tissue of a resistant and susceptible genotype of Brassica napus infected with Sclerotinia sclerotiorum. PLoS One 8:e65205.

14. Garg, H., Li, H., Sivasithamparam, K., Kuo, J., and Barbetti, M. J. 2010. The infection processes of Sclerotinia sclerotiorum in cotyledon tissue of a resistant and a susceptible genotype of Brassica napus. Ann. Bot. 106:897908.

15. Garg, H., Sivasithamparam, K., Banga, S. S., and Barbetti, M. J. 2008 Cotyledon assay as a rapid and reliable method of screening for resistance against Sclerotinia sclerotiorum in Brassica napus genotypes. Australas. Plant Pathol. 37:106-111.

16. Ge, X. T., Li, Y. P., Wan, Z. J., You, M. P., Finnegan, P. M., Banga, S. S., Sandhu, P. S., Garg, H., Salisbury, P. A., and Barbetti, M. J. 2012. Delineation of Sclerotinia sclerotiorum pathotypes using differential resistance responses on Brassica napus and B. juncea genotypes enables identification of resistance to prevailing pathotypes. Field Crops Res. 127:248-258.

17. Hegedus, D. D., and Rimmer, S. R. 2005. Sclerotinia sclerotiorum: When "to be or not to be" a pathogen? FEMS Microbiol. Lett. 251:177-184.

18. Hims, M. J. 1979. Damping-off of Brassica napus ('mustard and cress') by Sclerotinia sclerotiorum. Plant Pathol. 28:201-202.

19. Jurke, C. J., and Fernando, W. G. D. 2008. Comparison of growth room screening techniques for the determination of physiological resistance to Sclerotinia stem rot in Brassica napus. Arch. Phytopathol. Plant Prot. 41:157-174.

20. Kim, H. S., Hartman, G. L., Manandhar, J. B., Graef, G. L., Steadman, J. R., and Diers, B. W. 2000. Reaction of soybean cultivars to Sclerotinia stem rot in field, greenhouse, and laboratory evaluations. Crop Sci. 40:665-669.

21. Kim, W. G., and Cho, W. D. 2003. Occurrence of Sclerotinia rot in cruciferous crops caused by Sclerotinia spp. Plant Pathol. J. 19:69-74.

22. Kus, J. V., Zaton, K., Sarkar, R., and Cameron, R. K. 2002. Age-related resistance in Arabidopsis is a developmentally regulated defence response to Pseudomonas syringae. Plant Cell 14:479-490.

23. Laemmlen, F. 2001. Damping-off diseases. Publ. No. 8041. University of California, Davis

24. Leisner, S. M., Turgeon, R., and Howell, S. H. 1993. The effects of host plant development and genetic determinants on the long-distance movement of cauliflower mosaic virus in infected turnip plants. Plant Cell 5:191-202.

25. Li, C. X., Li, H., Siddique, A. B., Sivasithamparam, K., Salisbury, P. Banga, S. S., Banga, S., Chattopadhyay, C., Kumar, A., Singh, R., Singh, D., Agnihotri, A., Liu, S. Y., Li, Y. C., Tu, J., Fu, T. D., Wang, Y. F., and 
Barbetti, M. J. 2007. The importance of the type and time of inoculation and assessment in the determination of resistance in Brassica napus and $B$. juncea to Sclerotinia sclerotiorum. Aust. J. Agric. Res. 58:1198-1203.

26. Li, C. X., Li, H., Sivasithamparam, K., Fu, T. D., Li, Y. C., Liu, S. Y., and Barbetti, M. J. 2006. Expression of field resistance under Western Australian conditions to Sclerotinia sclerotiorum in Chinese and Australian Brassica napus and Brassica juncea germplasm and its relation with stem diameter. Aust. J. Agric. Res. 57:1131-1135.

27. Li, C. X., Liu, S. Y., Sivasithamparam, K., and Barbetti, M. J. 2009. New sources of resistance to Sclerotinia stem rot caused by Sclerotinia sclerotiorum in Chinese and Australian Brassica napus and B. juncea germplasm screened under Western Australian conditions. Australas. Plant Pathol. 38:149-152.

28. Li, H., Smyth, F., Barbetti, M. J., and Sivasithamparam, K. 2006. Relationship between Brassica napus seedling and adult plant responses to Leptosphaeria maculans is determined by plant growth stage at inoculation and temperature regime. Field Crops Res. 96:428-437.

29. Li, R., Rimmer, R., Buchwaldt, L., Sharpe, A. G., Séguin-Swartz, G., and Hegedus, D. D. 2004. Interaction of Sclerotinia sclerotiorum with Brassica napus: cloning and characterization of endo- and exo-polygalacturonases expressed during saprophytic and parasitic modes. Fungal Genet. Biol. 41:754-765.

30. Lumsden, R. D. 1979. Histology and physiology of pathogenesis in plant diseases caused by Sclerotinia species. Phytopathology 69:890-896.

31. McLaren, N. W., and Craven, M. 2008. Evaluation of soybean cultivars for resistance to Sclerotinia stalk rot in South Africa. Crop Prot. 27:231-235.
32. Mei, J., Qian, L., Disi, J., Yang, X., Li, Q., Li, J., Frauen, M., Cai, D., and Qian, W. 2011. Identification of resistant sources against Sclerotinia sclerotiorum in Brassica species with emphasis on B. oleracea. Euphytica 177:393-399.

33. Pedras, M. S. C., and Yaya, E. E. 2010. Phytoalexins from Brassicaceae: news from the front. Phytochemistry 71:1191-1197.

34. Rakow, G. 2004. Species origin and economic importance of Brassica. Pages 3-11 in: Brassica, vol. 54. E. C. Pua and C. Douglas, eds. SpringerVerlag, Berlin, Heidelberg, Germany.

35. Salisbury, P. A., and Barbetti, M. J. 2011. Breeding oilseed Brassica for climate change. Pages 448-463 in: Crop Adaptation to Climate Change. S S. Yadav, R. J. Redden, J. S. Hatfield, H. Lotze-Campen, and A. Hall, eds. John Wiley \& Sons, Ltd., Chichester, West Sussex, UK.

36. Singh, R., Singh, D., Li, H., Sivasithamparam, K., Yadav, N. R., Salisbury, P., and Barbetti, M. J. 2008. Management of Sclerotinia rot of oilseed Brassicas-a focus on India. Brassica 10:1-27.

37. Sylvester-Bradley, R., and Makepeace, R. J. 1984. A code for stages of development in oilseed rape (Brassica napus L.). Aspects Appl. Biol. 6:399419.

38. Uloth, M., You, M. P., Finnegan, P. M., Banga, S. S., Banga, S. K., Sandhu, P. S., Huang, Y., Salisbury, P., and Barbetti, M. J. 2013. New sources of resistance to Sclerotinia sclerotiorum for crucifer crops. Field Crops Res. $154: 40-52$

39. Zhao, J., and Meng, J. 2003. Genetic analysis of loci associated with partial resistance to Sclerotinia sclerotiorum in rapeseed (Brassica napus L.) Theor. Appl. Genet. 106:759-764. 